\section{PUVA-Badtherapie bei Necrobiosis lipoidica}

R. Schiener, S. C. Behrens-Williams, P. Gottlöber, H. Pillekamp, R. U. Peter, M. Kerscher

Abteilung Dermatologie, Universitätsklinikum Ulm (Ärztl. Direktor: Prof. Dr. R. U. Peter)
Zusammenfassung. Die Necrobiosis lipoidica resultiert unbehandelt aufgrund fortschreitenden Verlaufes und damit verbundener kosmetischer Beeinträchtigung häufig in starkem subjektiven Krankheitsgefühl. Bisher existiert jedoch kein allgemein anerkanntes Therapieverfahren. Die PUVA-Bad-Photochemotherapie hat sich in der Behandlung entzündlicher Dermatosen, etwa der Psoriasis vulgaris und des atopischen Ekzems, aber auch des Granuloma anulare, das der Necrobiosis lipoidica sehr nahe steht, sehr bewährt. Wir berichten über eine Patientin, bei der eine lange bestehende Necrobiosis lipoidica erfolgreich mit einer PUVA-Bad-Photochemotherapie behandelt werden konnte.

PUVA-Bath-Therapy of Necrobiosis Lipoidica. Necrobiosis lipoidica untreated often results in strong subjective complaints due to its progredience and cosmetic impairment. So far, no generally accepted therapy exists. PUVA-bath-therapy has shown to be effective in the treatment of inflammatory disorders of the skin such as psoriasis vulgaris, atopic dermatitis and also in granuloma anulare, which is considered to be close to necrobiosis lipoidica. We report a case of longlasting necrobiosis lipoidica, which has been successfully treated with PUVA-bath-therapy.

Die Necrobiosis lipoidica ist eine ätiologisch bisher unklare chronische Hauterkrankung, die aufgrund teils erheblicher kosmetischer Beeinträchtigung für viele Patienten einen hohen Krankheitswert hat und für die bis heute kein allgemein anerkanntes Therapieverfahren existiert. Die topische, teils okklusive Anwendung externer Kortikosteroide, auch in Verbindung mit Kompressionsverbänden, kann die Progredienz der Erkrankung verhindern und in Einzelfällen zur Abheilung führen [1-3]. Therapieversuche mit Pentoxifyllin oder hochdosiertem Nikotinsäureamid erwiesen sich in Einzelfällen als erfolgreich [4 - 7]. Der Einsatz von Clofazimin kann in nichtexponierten Arealen zu reversiblen, jedoch störenden rötlichbraunen Pigmentierungen führen [8]. In schweren Fällen ist der Einsatz systemischer Kortikosteroide möglich, bei der häufigen Assoziation mit einem Diabetes mellitus ist jedoch eine

Akt Dermatol 2001; 27: 194-197

(c) Georg Thieme Verlag Stuttgart · New York ISSN 0340-2541 strenge Indikationsstellung geboten. Die PUVA-Bad-Photochemotherapie wird bisher sehr erfolgreich bei Psoriasis vulgaris, atopischem Ekzem, palmoplantaren Dermatosen, Mastozytosen, zirkumskripter Sklerodermie, Lichen ruber sowie dem Granuloma anulare eingesetzt [9-15]. Wir stellen hier eine 36-jährige Patientin mit seit 4 Jahren bestehender, therapieresistenter Necrobiosis lipoidica vor, bei der wir uns aufgrund der Persistenz und Progredienz der Hautveränderungen über 4 Jahre, einer ausgesprochenen Therapieresistenz sowie aufgrund unserer positiven Erfahrungen in der Therapie des Granuloma anulare zur Durchführung einer PUVA-Bad-Photochemotherapie entschieden.

\section{Fallbeschreibung}

\section{Anamnese}

Bei der 36-jährigen Patientin traten 1995 erstmalig erythematöse Makulae an beiden Unterschenkeln streckseitenbetont auf. Die gelegentlich juckenden Hautveränderungen zeigten ein progredientes Flächenwachstum. Externe Kortikosteroide blieben auch unter konsequenter Kompressionstherapie ohne Erfolg, eine systemische Kortikoidtherapie sowie die Gabe von Pentoxifyllin konnten die Hauterscheinungen ebenfalls nicht bessern.

\section{Befund}

An den Unterschenkelstreckseiten zeigten sich mehrere unregelmäßig konfigurierte, scharf begrenzte, teils konfluierende atrophische Herde mit braungelbem, sklerotischem Zentrum, teils von Teleangiektasien durchzogen. Die Herde waren von einem entzündlichen, erythematösen Randsaum umgeben (Abb. 1 a).

Labor

BKS auf 26/55 erhöht. Blutbild, Leber- und Nierenwerte sowie Glukose- und Eiweißstoffwechsel waren normwertig. Weiterhin normwertiges ACE, kein Nachweis antinukleärer Antikörper. Negative Borrelien- und Hepatitisserologie. Kein Nachweis säurefester Stäbchen im Hautbiopsat. Die internistisch orientierende Untersuchung ergab keine pathologischen Auffälligkeiten, insbesondere keinen Anhalt für das Vorliegen eines Diabetes mellitus bei unauffälligem oralen Glukosetoleranztest. 

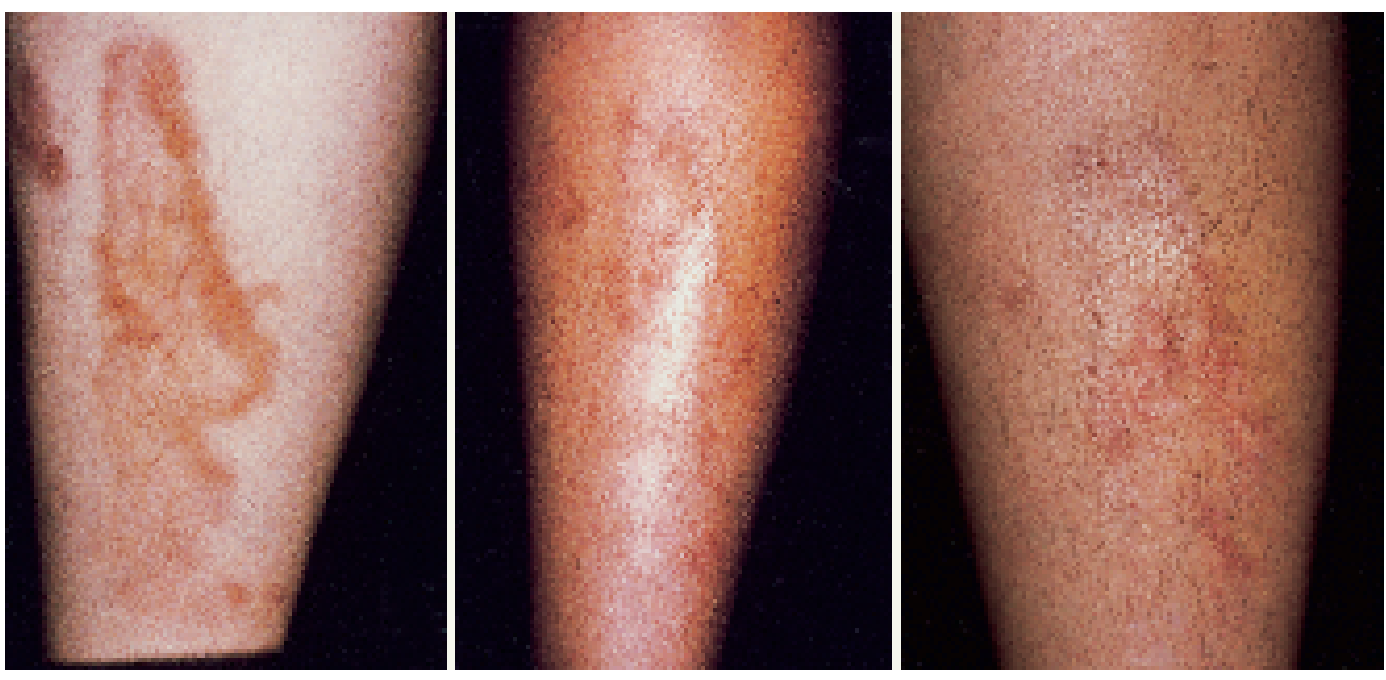

Abb. 1 Necrobiosis lipoidica. a Scharf begrenzter atrophischer Herd mit braungelbem, sklerotisch hartem, teils von Teleangiektasien durchzogen und entzündlichem, erythematösem Randsaum vor PUVA-Bad-Photochemotherapie. b Unter PUVABad-Photochemotherapie. c 3 Monate nach Therapieende.

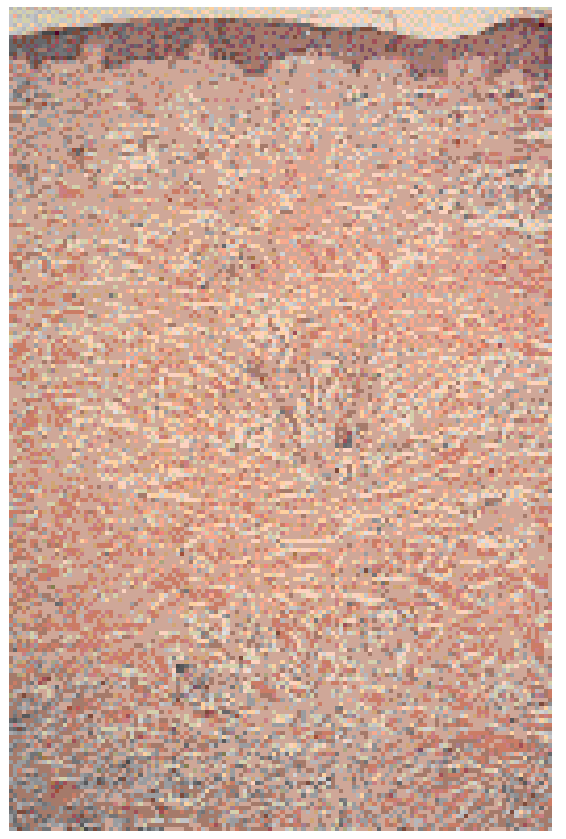

Abb. 2 Histologie: Teils degenerative Kollagenbezirke, teils breitere Kollagenbündel sowie Infiltrate aus histiozytären Zellen, Lymphozyten und einzelnen mehrkernigen Zellen (HE, ×30).

\section{Histologie}

Bis in die tiefere Dermis finden sich Infiltrate aus histiozytären Zellen, Lymphozyten und einzelnen mehrkernigen Histiozyten. Vereinzelter Nachweis nekrobiotischer Kollagenareale sowie degenerativer Kollagenbezirke und teils breiterer Kollagenbündel (Abb. 2).

\section{Phlebologie}

Die lichtreflexionsrheographischen sowie dopplersonographischen Untersuchungen ergaben keinen Hinweis auf das Vorliegen einer Insuffizienz des oberflächlichen Venensystems oder einer relevanten Perforansinsuffizienz.

\section{0-MHz-Sonographie}

Im Vergleich zur gesunden Haut zeigte sich ein deutlich verbreitertes Korium mit verminderter Echogenität in den mittleren und tiefen Koriumanteilen (Hautdicke 2,4 mm, Dichte 15). In der Subkutis finden sich bis an die Muskelfaszie reichend vermehrt echoreiche Strukturen, Korium und Subkutis sind kaum voneinander abgrenzbar (Abb. 3 a).

\section{Therapie und Verlauf}

Aufgrund des starken Leidensdruckes entschlossen wir uns zur Durchführung einer Teilkörper-PUVA-Bad-Photochemotherapie beider Unterschenkel. Unmittelbar nach zwanzigminütigem Warmwasserbad $\left(37^{\circ} \mathrm{C}\right)$ mit Zusatz von 8-MOP in einer Konzentration von $0,5 \mathrm{mg} / \mathrm{l}$ erfolgte die UVA-Bestrahlung. Die initiale UVA-Dosis betrug $0,3 \mathrm{~J} / \mathrm{cm}^{2}$ und wurde frühestens nach jeder zweiten Therapie um maximal $0,3 \mathrm{~J} / \mathrm{cm}^{2}$ gesteigert. Die Bestrahlung erfolgte 3-mal wöchentlich. Unter dieser Therapie kam es nicht zum Auftreten einer phototoxischen Reaktion. Bereits nach 16 Sitzungen zeigte sich klinisch wie sonographisch eine deutliche Befundbesserung. Der entzündliche Randsaum war deutlich abgeblasst, die atrophischen zentralen Areale nur noch schwach abgrenzbar. Nach 52 Behandlungen zeigte sich 20-MHz-sonographisch eine deutliche Zunahme der Echogenität des Koriums (Abb. 3 b). Die initial mit 2,4 mm deutlich vermehrte Hautdicke lag mit $1,4 \mathrm{~mm}$ in der Norm, die Dichte betrug 33 (hauterscheinungsfreie korrespondiernde Kontrolle: Hautdicke 1,4 mm, Dichte 35). Klinisch palpatorisch zeigte sich eine normale Konsistenz der Haut mit deutlicher Abblassung des Randsaumes (Abb. 1 b).

\section{Besprechung}

Die Necrobiosis lipoidica ist eine chronisch verlaufende, granulomatös entzündliche Hauterkrankung, die vorzugsweise bei Frauen im mittleren Lebensalter auftritt und aufgrund kosmetischer Beeinträchtigungen in starkem Leidensdruck resultieren kann. In etwa 40\% der Fälle kann die Necrobiosis lipoidica mit einem Diabetes mellitus assoziiert sein. Klinisch finden sich unregelmäßig konfigurierte, scharf begrenzte, teils konfluierende, atrophische Herde mit gelbem bis braungelbem, sklerotischem Zentrum, das von Teleangiektasien durchzogen 

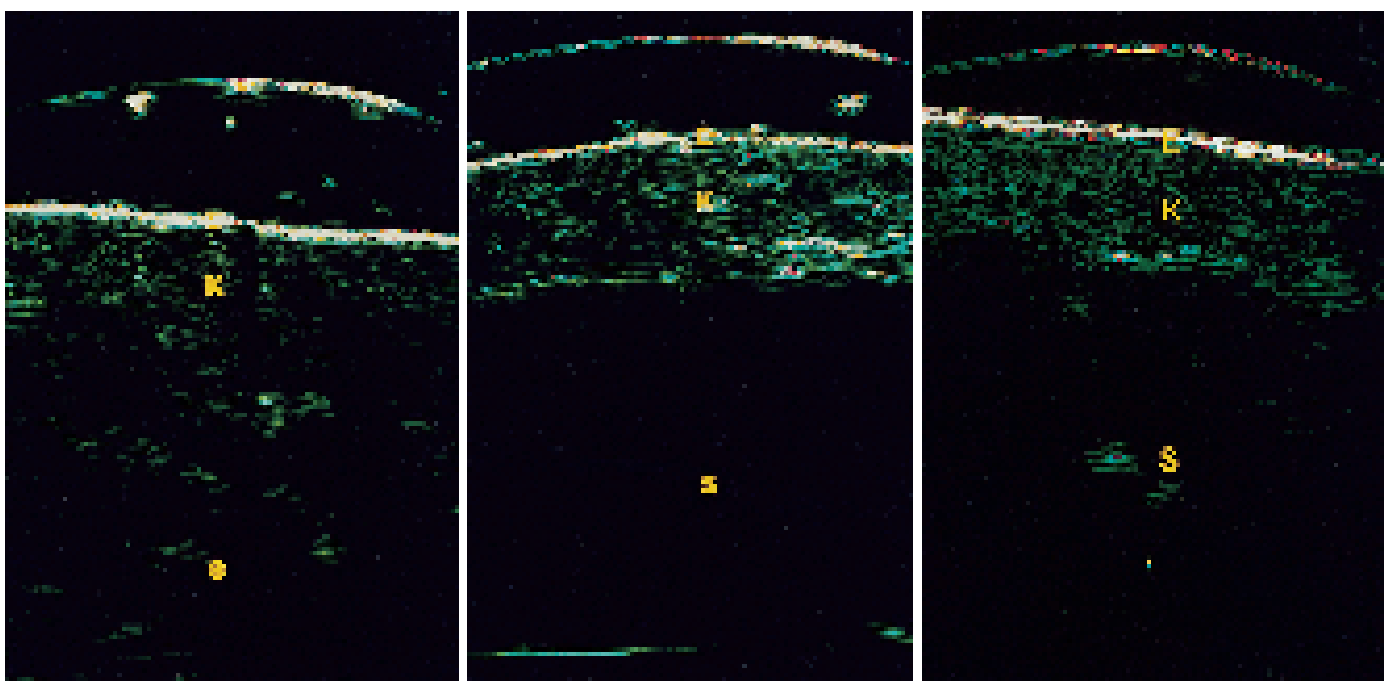

Abb. 3 Necrobiosis lipoidica 20-MHz-Sonographie (DUB 20). a Deutlich verbreitertes Korium mit verminderter Echogenität in den mittleren und tiefen Koriumanteilen. Vermehrt echoreiche Strukturen in der Subkutis, Trennung zwischen Korium und Subkutis nur schwer möglich. b Normalisierte Koriumdicke und -dichte nach PUVA-BadPhotochemotherapie. c Normalbefund 3 Monate nach Therapieende.

ist. Die Herde sind meist von einem rötlich-violetten Randsaum umgeben. Prädilektionsstellen sind die Streckseiten der Unterschenkel, Fußrücken sowie die Fußgelenkregion. Histologisch imponieren in der oberen, mittleren und tiefen Dermis nekrobiotische Kollagenareale mit Lipidablagerungen, histiozytäre Infiltrate, zum Teil in Palisadenstellung, sowie Riesenzellen.

Aufgrund der erfolgreichen Anwendung der Bad-Photochemotherapie bei anderen entzündlichen Hauterkrankungen, wie auch dem Granuloma anulare, und der sehr guten WirkungsNebenwirkungsrelation dieser Therapiemodalität, behandelten wir unsere Patientin mit therapieresistenter Necrobiosis lipoidica mit einer PUVA-Bad-Photochemotherapie. Nach insgesamt 52 Behandlungen kam es bei einer kumulativen Dosis von $84,5 \mathrm{~J} / \mathrm{cm}^{2}$ zu einer nahezu vollständigen Abheilung der Läsionen. Es traten keine phototoxischen Reaktionen auf. Die Patientin blieb über einen Nachbeobachtungszeitraum von 3 Monaten rezidivfrei (Abb.1 c, 3c).

Die bei unserer Patientin für den Therapieerfolg erforderliche Bestrahlungsanzahl und kumulative UVA-Dosis entspricht sehr gut den von Salomon et al. publizierten Daten, die in einer Studie zur PUVA-Bad-Therapie des Granuloma anulare über gute therapeutische Erfolge nach durchschnittlich 55 Therapiesitzungen bei einer mittleren kumulativen UVA-Dosis von $69,5 \mathrm{~J} / \mathrm{cm}^{2}$ berichteten [16].

Da die Serumkonzentrationen von 8-MOP im Rahmen der PUVA-Bad-Photochemotherapie unterhalb der Nachweisgrenze liegen, entfallen die bei der oralen PUVA-Therapie häufig auftretenden gastrointestinalen Nebenwirkungen, zudem sind weiterreichende Schutzmaßnahmen nicht erforderlich, weil nicht gebadete Hautareale keine erhöhte Photosensitivität aufweisen und die Photosensitivität gebadeter Hautareale rasch abklingt $[17,18]$. Mit der PUVA-Bad-Photochemotherapie steht somit ein auch ambulant gut anwendbares, nebenwirkungsarmes, bei unserem Patienten sehr wirksames Therapieverfahren der Necrobiosis lipoidica zur Verfügung, das einen hohen therapeutischen Index aufweist.

\section{Literatur}

${ }^{1}$ Goette DK. Resolution of necrobiosis lipoidica with exclusive clobetasol propionate treatment. J Am Acad Dermatol 1990; 22: $855-856$

${ }^{2}$ Lakhanpal S, Ginsburg WW, Michet CJ, Doyle JA, Moore SB. Eosinophilic fasciitis: clinical spectrum and therapeutic response in 52 cases. Semin Arthritis Rheum 1988; 17: 221 - 231

${ }^{3}$ Volden G. Successful treatment of chronic skin diseases with clobetasol propionate and a hydrocolloid occlusive dressing. Acta Derm Venereol 1992; 72: 69-71

${ }^{4}$ Espana A, Sanchez Yus E, Serna MJ, Redondo P, Robledo A, Quintanilla E. Chronic balanitis with palisading granuloma: an atypical genital localization of necrobiosis lipoidica responsive to pentoxifylline. Dermatology 1994; 188: 222-225

${ }^{5}$ HandfieldJones S, Jones S, Peachey R. High dose nicotinamide in the treatment of necrobiosis lipoidica. Br J Dermatol 1988; 118: $693-696$

${ }^{6}$ Littler CM, Tschen EH. Pentoxifylline for necrobiosis lipoidica diabeticorum. J Am Acad Dermatol 1987; 17: 314-316

${ }^{7}$ Rubel DM, Wood G, Rosen R, JoppMcKay A. Generalised granuloma annulare successfully treated with pentoxifylline. Australas J Dermatol 1993; 34: 103-108

${ }^{8}$ Mensing H. Clofazimine - therapeutische Alternative bei Necrobiosis lipoidica und Granuloma anulare. Hautarzt 1989; 40: 99103

${ }^{9}$ Collins P, Rogers S. Bath-water compared with oral delivery of 8methoxypsoralen PUVA therapy for chronic plaque psoriasis. Br J Dermatol 1992; 127: $392-395$

${ }^{10}$ Godt O, Proksch E, Streit V, Christophers E. Short- and long-term effectiveness of oral and bath PUVA therapy in urticaria pigmentosa and systemic mastocytosis. Dermatology 1997; 195: 35 - 39

${ }^{11}$ Kerscher M, Volkenandt M, Lehmann P, Plewig G, Röcken M. PUVA-bath photochemotherapy of lichen planus. Arch Dermatol 1995; 131: 1210-1211

${ }^{12}$ Kerscher M, Meurer M, Sander C, Volkenandt M, Lehmann P, Plewig G, Röcken M. PUVA bath photochemotherapy for localized scleroderma. Evaluation of 17 consecutive patients. Arch Dermatol 1996; 132: 1280-1282

${ }^{13}$ Langrock A, Weyers W, Schill WB. Balneophotochemotherapie bei disseminiertem Granuloma anulare. Hautarzt 1998; 49: $303-$ 306 
${ }^{14}$ Behrens S, von Kobyletzki G, Gruss C, Reuther T, Altmeyer P, Kerscher M. PUVA-bath photochemotherapy (PUVA-soak therapy) of recalcitrant dermatoses of the palms and soles. Photodermatol Photoimmunol Photomed 1999; 15: 47-51

${ }^{15}$ von Kobyletzki G, Gruss C, Altmeyer P, Kerscher M. Balneophotochemotherapie des Lichen ruber. Eigene Ergebnisse und Vergleich mit bisher angewandten Photochemotherapiemodalitäten. Hautarzt 1997; 48: $323-327$

${ }^{16}$ Salomon N, Walchner M, Messer G, Plewig G, Rocken M. Bade-PUVA-Therapie bei Granuloma anulare. Hautarzt 1999; 50: 275 279

${ }^{17}$ Gruss C, Behrens S, Reuther T, Husebo L, Neumann N, Altmeyer P, Lehmann P, Kerscher M. Kinetics of photosensitivity in bath-PUVA photochemotherapy. J Am Acad Dermatol 1998; 39: 443-446

${ }^{18}$ Neumann NJ, Ruzicka T, Lehmann P, Kerscher M. Rapid decrease of phototoxicity after PUVA bath therapy with 8-methoxypsoralen. Arch Dermatol 1996; 132: 1394

\section{Dr. Ralf Schiener}

Universitätsklinikum Ulm Abteilung Dermatologie

Oberer Eselsberg 40

89081 Ulm 